\title{
Automatic insulin-pump suspension reduces hypoglycaemia
}

A novel 'threshold-suspend' feature of insulin pumps can safely and effectively reduce hypoglycaemia in patients with with type 1 diabetes mellitus (T1DM), a new study published in The New England Journal of Medicine reports.

Hypoglycaemia is a common adverse effect of insulin therapy in people with T1DM, particularly during the night. Sensor-augmented insulin pumps, which integrate pumps with continuous glucose monitoring, provide a more effective therapy than multiple daily insulin injections. However, these pumps have not yet been proven to reduce the risk of hypoglycaemia.

Bergenstal et al. investigated sensoraugmented insulin pumps containing a new threshold-suspend feature that automatically suspends basal insulin delivery when sensor glucose values reach a pre-set low level $(\leq 3.9 \mathrm{mmol} / \mathrm{l})$.

In a randomized controlled 3-month study, the researchers assigned patients to receive sensor-augmented insulin-pump therapy either with $(n=121)$ or without $(n=126)$ this feature. Patients were aged 16-70 years, had been diagnosed as having T1DM $>2$ years prior to the study and had documented

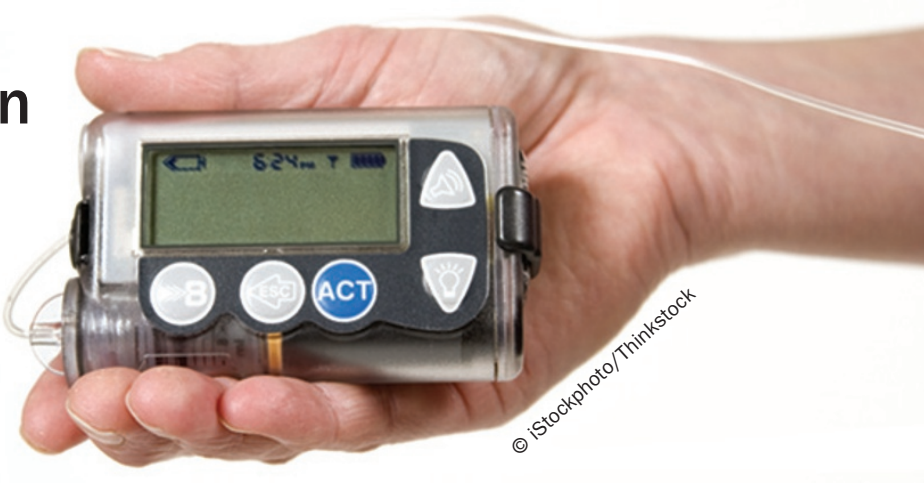
nocturnal hypoglycaemia (that is, a drop in sensor glucose values to $\leq 3.6 \mathrm{mmol} / \mathrm{l}$ for at least $20 \mathrm{~min}$ between $10.00 \mathrm{pm}$ and $8.00 \mathrm{am})$.

The frequency, magnitude and duration of hypoglycaemic events were used to compare the efficacy of the two different insulin pumps. Patients in the thresholdsuspend group had significantly lower weekly rates of night-time hypoglycaemic events than the control group, and the rate of nocturnal hypoglycaemic events decreased by $31.8 \%$ in the thresholdsuspend group compared with the control group. Moreover, the severity and duration of hypoglycaemic events was reduced in patients using an insulin pump with the threshold-suspend feature. No change in
$\mathrm{HbA}_{1 c}$ level occurred in either group, and no adverse events were reported in the threshold-suspend group, whereas four patients in the control group experienced severe hypoglycaemia.

The authors conclude that the threshold-suspend feature of sensoraugmented insulin pumps can safely reduce the frequency of hypoglycaemia, both diurnal and nocturnal, without any loss in overall glycaemic control.

Elisabeth Kugelberg

Original article Bergenstal, R. M. et al. Threshold-based insulin-pump interruption for reduction of hypoglycemia. N. Engl. J. Med. doi:10.1056/NEJMoa1303576 